\title{
EXACT SUPPORT RECOVERY FOR LINEAR INVERSE PROBLEMS WITH SPARSITY CONSTRAINTS*
}

\author{
DENNIS TREDE ${ }^{\dagger}$
}

\begin{abstract}
There are a couple of methods with sparsity enhancing properties for regularization of inverse problems. This paper deals with the Tikhonov regularization with an $\ell^{1}$ penalty and with the orthogonal matching pursuit. Conditions are derived, which ensure that the regularized solution has the exact support. These conditions are especially applicable in the case of ill-posed problems, where other conditions based on coherence or the restricted isometry property are usually not applicable. Surprisingly, the conditions coincide for both, for the $\ell^{1}$-penalized Tikhonov regularization and for the orthogonal matching pursuit.
\end{abstract}

Key words. Inverse problems, ill-posed problems, sparsity constraints, exact recovery, Tikhonov regularization, greedy algorithm, orthogonal matching pursuit.

AMS subject classifications. Primary 47A52, 65J20; Secondary 94A12.

1. Introduction. In this paper we consider linear inverse problems with a bounded injective operator $K: \ell^{2} \rightarrow \mathcal{H}$ between the sequence space $\ell^{2}$ and a separable Hilbert space $\mathcal{H}$,

$$
K u=g .
$$

In the following, let $\left\{e_{j}\right\}_{j \in \mathbb{N}}$ denote the standard basis of $\ell^{2}$. We additionally assume that the operator norm of $K$ is bounded by 1 . The assumption is a common condition for linear inverse problems and, actually, this is not really a limitation, because every inverse problem $K u=g$ with bounded operator $K$ can be rescaled so that $\|K\| \leq 1$ holds.

We are given a noisy observation $g^{\varepsilon}=g+\eta \in \mathcal{H}$ with noise level $\left\|g-g^{\varepsilon}\right\|=\|\eta\| \leq \varepsilon$ and try to reconstruct the solution $u \in \ell^{2}$ of $K u=g$ from the knowledge of $g^{\varepsilon}$. We assume that the operator equation $K u=g$ has a solution $u^{\diamond}$ that is finitely supported, i.e.

$$
u^{\diamond} \in \ell^{0}
$$

There are several methods utilizing this prior knowledge for the reconstruction. In the following we introduce two examples of regularization methods incorporating sparsity constraints, namely,

i) the Tikhonov regularization with sparsity constraints, which is a variational regularization methods, and

ii) the orthogonal matching pursuit, which is an iterative regularization method. In this paper we investigate error estimates for these two methods, namely, we deduce conditions which ensure that the approximate solution has the same support as $u^{\diamond}$.

In [3], the Tikhonov regularization with $\ell^{1}$-norm penalty has been introduced in the infinite dimensional inverse problems setting to obtain a sparse minimizer. More precisely, given a regularization parameter $\alpha>0$ in [3] the authors introduce the

\footnotetext{
*Received October 26, 2010; accepted for publication February 7, 2011.

$\dagger$ †entrum für Technomathematik, Universität Bremen, Fachbereich Mathematik/Informatik, Postfach 3304 40, D-28334 Bremen, Germany (trede@math.uni-bremen.de). The author was supported by the BMBF project INVERS by Grant 03MAPAH7.
} 
following functional

$$
T_{\alpha}(u)=\left\|K u-g^{\varepsilon}\right\|_{\mathcal{H}}^{2}+\alpha\|u\|_{1} .
$$

In contrast to the classical Tikhonov functional with a quadratic penalty, the $\ell^{1}$ penalized functional (1.2) promotes sparsity since small coefficients are penalized stronger. This functional has also been used in the context of sparse recovery under the name basis pursuit denoising [2].

The minimization of the $\ell^{1}$-penalized Tikhonov functional is not the subject of this paper, for a comparison of some recent algorithms see e.g. [14]. Here, we assume that a suitable minimization method is available, and we investigate error estimates for the minimizer

$$
u^{\alpha, \varepsilon}:=\arg \min T_{\alpha}(u) .
$$

Daubechies et al. [3] showed that the minimization of (1.2) is indeed a regularization while error estimates and convergence rates have been derived by Lorenz [12] and Grasmair et al. [10] under additional source conditions. In this paper we derive conditions ensuring that the minimizer $u^{\alpha, \varepsilon}=\arg \min T_{\alpha}(u)$ has the same support as $u^{\diamond}$. In the context of sparse recovery, this phenomenon is called exact recovery and in that context such conditions have been proved in $[7,8,21]$. Unless the set $\left\{K e_{i}\right\}_{i \in \mathbb{N}}$ is close to the singular vectors of the operator $K$, the concepts from the field of sparse recovery in general are not applicable and one has to resort to other conditions $[6,11]$. In this paper we cite the conditions from [13], which work for ill-posed linear operator equations.

The second sparsity enforcing method we analyze in this paper - the orthogonal matching pursuit (OMP) - is an iterative method. To given data $g^{\varepsilon}$ and using the initial guess $u^{0, \varepsilon}=0$, we minimize the residual $\left\|K \cdot-g^{\varepsilon}\right\|_{\mathcal{H}}$ "greedily". OMP was first proposed in the signal processing context by Davis et al. in [15] and Pati et al. in [18]. Algorithmically, it can be written as follows:

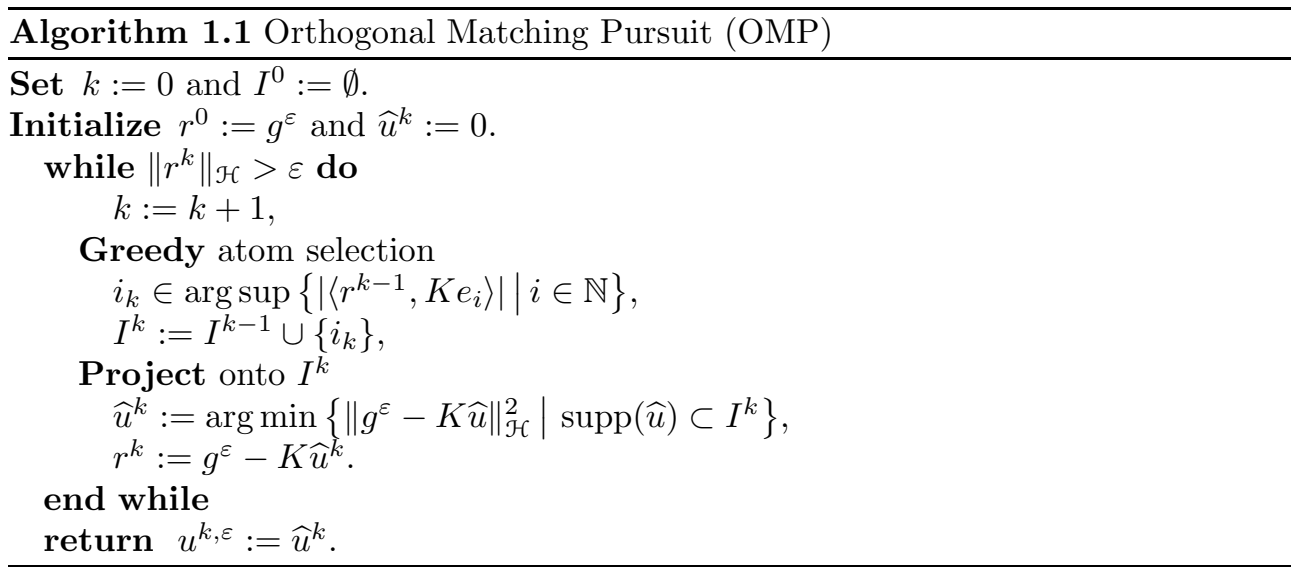

In [4] regularizing properties and convergence rates for OMP have been deduced on certain conditions. In this paper we deal with conditions which ensure the recovery of the exact support $I$ with OMP, and we give an error estimate of the approximate solution. Some analysis in this sense is already available in a sparse approximation setting, cf. e.g. $[20,5]$. As for the $\ell^{1}$-penalized Tikhonov regularization, the concept 
of sparse approximation cannot be transfered to ill-posed inverse problems and one has to impose other conditions $[6,11,4]$.

The paper is organized as follows. In section 2 we formulate two conditions that ensure the exact recovery of the support of $u^{\diamond}$. These conditions are valid, both for the $\ell^{1}$-Tikhonov regularization and for OMP. In sections 3 we give the proofs for the conditions from section 2 for the orthogonal matching pursuit. The proofs for $\ell^{1}$ penalized Tikhonov regularization have been done in $[19,13]$. In section 4 we give a conclusion on exact recovery conditions for inverse problems with sparsity constraints and make possible forecasts on the future work.

2. Conditions for exact support recovery. We give two conditions which ensure that the unknown support of the sparse solution $u^{\diamond} \in \ell^{0}$ is recovered exactly, both for the $\ell^{1}$-penalized Tikhonov functional and for OMP. With $I$ we denote the support of $u^{\diamond}$, i.e.

$$
I:=\operatorname{supp}\left(u^{\diamond}\right):=\left\{i \in \mathbb{N} \mid u_{i}^{\diamond} \neq 0\right\} .
$$

To formulate the statements, we need the following notations. For a subset $J \subset \mathbb{N}$, we denote with $P_{J}: \ell^{2} \rightarrow \ell^{2}$ the projection onto $J$,

$$
P_{J} u:=\sum_{j \in J} u_{j} e_{j},
$$

i.e. the coefficients $j \notin J$ are set to 0 and hence $\operatorname{supp}\left(P_{J} u\right) \subset J$. With that definition $K P_{J}: \ell^{2} \rightarrow \mathcal{H}$ denotes the operator $K$ restricted to $J$. Moreover, for a linear operator $B$ we denote the pseudoinverse operator by $B^{\dagger}$. With these definitions we are able to formulate the first condition for exact recovery.

THEOREM 2.1 (Exact recovery condition in the presence of noise). Let $u^{\diamond} \in \ell^{0}$ with $\operatorname{supp}\left(u^{\diamond}\right)=I$ and $g^{\varepsilon}=K u^{\diamond}+\eta$ the noisy data with noise-to-signal ratio

$$
r_{\eta / u}:=\frac{\sup _{i \in \mathbb{N}}\left|\left\langle\eta, K e_{i}\right\rangle\right|}{\min _{i \in I}\left|u_{i}^{\diamond}\right|} .
$$

Assume that the operator norm of $K$ is bounded by 1 and that $K$ is injective. Then the exact recovery condition in the presence of noise $(\varepsilon \mathrm{ERC})$

$$
\sup _{i \in I^{\mathrm{C}}}\left\|\left(K P_{I}\right)^{\dagger} K e_{i}\right\|_{\ell^{1}}<1-2 r_{\eta / u}\left\|\left(P_{I} K^{*} K P_{I}\right)^{-1}\right\|_{\ell^{1}, \ell^{1}}
$$

ensures that both, the $\ell^{1}$-penalized Tikhonov regularization and OMP recover the support I exactly.

Remark 2.2. For the $\ell^{1}$-penalized Tikhonov regularization the condition (2.1) ensures that there exists a regularization parameter $\alpha>0$ which allows exact recovery of the support, see [13].

Due to the expressions $\left\|\left(P_{I} K^{*} K P_{I}\right)^{-1}\right\|_{\ell^{1}, \ell^{1}}$ and $\sup _{i \in I^{\mathrm{C}}}\left\|\left(K P_{I}\right)^{\dagger} K e_{i}\right\|_{\ell^{1}}$, the $\varepsilon \mathrm{ERC}(2.1)$ is hard to evaluate, especially since the support $I$ is unknown. Therefore, we follow $[6,11]$ and give a weaker sufficient recovery condition, that depends on inner products of images of $K$ restricted to $I$ and $I^{\complement}$. According to [11,6], for the sake of an easier presentation we define

$$
\mathrm{COR}_{I}:=\sup _{i \in I} \sum_{\substack{j \in I \\ j \neq i}}\left|\left\langle K e_{i}, K e_{j}\right\rangle\right| \quad \text { and } \quad \operatorname{COR}_{I^{\mathrm{C}}}:=\sup _{i \in I^{\mathrm{C}}} \sum_{j \in I}\left|\left\langle K e_{i}, K e_{j}\right\rangle\right| .
$$


THEOREM 2.3 (Neumann exact recovery condition in the presence of noise). Let $u^{\diamond} \in \ell^{0}$ with $\operatorname{supp}\left(u^{\diamond}\right)=I$ and $g^{\varepsilon}=K u^{\diamond}+\eta$ the noisy data with noise-to-signal ratio $r_{\eta / u}$. Assume that the operator norm of $K$ is bounded by 1 and that $K$ is injective. Then the Neumann exact recovery condition in the presence of noise (Neumann $\varepsilon$ ERC)

$$
\mathrm{COR}_{I}+\mathrm{COR}_{I^{\mathrm{C}}}<\min _{i \in I}\left\|K e_{i}\right\|_{\mathcal{H}}^{2}-2 r_{\eta / u}
$$

ensures that both, the $\ell^{1}$-penalized Tikhonov regularization and OMP recover the support I exactly.

To apply the Neumann $\varepsilon$ ERC (2.2) one has to know the support $I$. However, with a certain prior knowledge the correlations $\mathrm{COR}_{I}$ and $\mathrm{COR}_{I^{\mathrm{c}}}$ can be estimated from above a priori, especially when the support $I$ is not known exactly. That way it is possible to obtain a priori computable conditions for exact recovery. In [4], this is done exemplarily for an example from mass spectrometry and for an example from digital holography of particles. This shows the practical relevance of the condition.

REMARK 2.4. By assumption, the operator norm of $K$ is bounded by 1, i.e. $\left\|K e_{i}\right\| \leq 1$ for all $i \in \mathbb{N}$. Hence to ensure the Neumann $\varepsilon E R C$ (2.2), one has necessarily for the noise-to-signal ratio $r_{\eta / u}<1 / 2$.

REMARK 2.5 (Exact recovery condition in terms of coherence). For sparse approximation problems, exact recovery is often characterized with the so-called coherence parameter $\mu$, which is defined by

$$
\mu:=\sup _{i \neq j}\left|\left\langle K e_{i}, K e_{j}\right\rangle\right| .
$$

Sometimes the so-called cumulative coherence $\mu_{1}$ is used, too, which for a positive integer $m$ is defined by

$$
\mu_{1}(m):=\sup _{\substack{\Lambda \subset \mathbb{N} \\|\Lambda|=m}} \sup _{i \notin \Lambda} \sum_{j \in \Lambda}\left|\left\langle K e_{i}, K e_{j}\right\rangle\right| .
$$

We remark that the correlations $\mathrm{COR}_{I}$ and $\mathrm{COR}_{I^{\mathrm{C}}}$ can be estimated from above, with $N:=\left\|u^{\diamond}\right\|_{\ell^{0}}$, by

$$
\begin{aligned}
\mathrm{COR}_{I} & =\sup _{i \in I} \sum_{\substack{j \in I \\
j \neq i}}\left|\left\langle K e_{i}, K e_{j}\right\rangle\right| \leq \mu_{1}(N-1) \leq(N-1) \mu, \\
\mathrm{COR}_{I^{\mathrm{C}}} & =\sup _{i \in I^{\mathrm{C}}} \sum_{j \in I}\left|\left\langle K e_{i}, K e_{j}\right\rangle\right| \leq \mu_{1}(N) \leq N \mu .
\end{aligned}
$$

With that, exact recovery conditions in terms of the coherence parameter $\mu$ and the cumulative coherence $\mu_{1}$ can easily be deduced from condition (2.2).

3. Proof of the ERCs for the orthogonal matching pursuit. Similar conditions for OMP have been proven in [4]. However, the corresponding condition from [4] is weaker than the $\varepsilon$ ERC (2.1) presented in this paper. For the proof we follow the techniques from [4].

Proof of theorem 2.1. We do the proof by induction. Assume that OMP recovered the correct patterns in the first $k$ steps, i.e.

$$
\widehat{u}^{k}=\sum_{i \in I^{k}} \widehat{u}_{i}^{k} e_{i}
$$


with $I^{k} \subset I$. Then we get for the residual

$$
\begin{aligned}
r^{k}: & =g^{\varepsilon}-K \widehat{u}^{k}=g+\eta-K \widehat{u}^{k}=K\left(\sum_{i \in I}\left(u_{i}^{\diamond}-\widehat{u}_{i}^{k}\right) e_{i}\right)+\eta \\
& =\sum_{i \in I}\left(u_{i}^{\diamond}-\widehat{u}_{i}^{k}\right) K e_{i}+\eta
\end{aligned}
$$

hence the noiseless residual $s^{k}:=r^{k}-\eta=\sum\left(u_{i}^{\diamond}-\widehat{u}_{i}^{k}\right) K e_{i}$ has support $I$. Following [4], we get the condition

$$
\sup _{i \in I^{\mathrm{C}}}\left\|\left(K P_{I}\right)^{\dagger} K e_{i}\right\|_{\ell^{1}}<1-2 \frac{\sup _{i \in \mathbb{N}}\left|\left\langle\eta, K e_{i}\right\rangle\right|}{\left\|P_{I} K^{*} s^{k}\right\|_{\ell^{\infty}}},
$$

which ensures a right choice in the $(k+1)$-st step. The last thing we have to afford to finish the proof is an estimation for the term $\left\|P_{I} K^{*} s^{k}\right\|_{\ell \infty}$ from below.

Since $K$ is injective, we get

$$
\begin{aligned}
\left\|P_{I} K^{*} s^{k}\right\|_{\ell^{\infty}} & \geq \frac{1}{\left\|\left(P_{I} K^{*} K P_{I}\right)^{-1}\right\|_{\ell \infty, \ell \infty}}\left\|\left(P_{I} K^{*} K P_{I}\right)^{-1} P_{I} K^{*} s^{k}\right\|_{\ell \infty} \\
& =\frac{1}{\left\|\left(P_{I} K^{*} K P_{I}\right)^{-1}\right\|_{\ell^{\infty}, \ell^{\infty}}}\left\|\left(P_{I} K^{*} K P_{I}\right)^{-1} P_{I} K^{*} K P_{I}\left(\sum\left(u_{i}^{\diamond}-\widehat{u}_{i}^{k}\right) e_{i}\right)\right\|_{\ell \infty} \\
& =\frac{1}{\left\|\left(P_{I} K^{*} K P_{I}\right)^{-1}\right\|_{\ell^{\infty}, \ell^{\infty}}} \max _{i \in I}\left|u_{i}^{\diamond}-\widehat{u}_{i}^{k}\right| .
\end{aligned}
$$

Since it holds that

$$
\max _{i \in I}\left|u_{i}^{\diamond}-\widehat{u}_{i}^{k}\right| \geq \max _{i \in I, i \notin I^{k}}\left|u_{i}^{\diamond}-\widehat{u}_{i}^{k}\right|=\max _{i \in I, i \notin I^{k}}\left|u_{i}^{\diamond}\right| \geq \min _{i \in I}\left|u_{i}^{\diamond}\right|,
$$

and since $\left\|\left(P_{I} K^{*} K P_{I}\right)^{-1}\right\|_{\ell^{\infty}, \ell^{\infty}}=\left\|\left(P_{I} K^{*} K P_{I}\right)^{-1}\right\|_{\ell^{1}, \ell^{1}}$, we get

$$
\left\|P_{I} K^{*} s^{k}\right\|_{\ell^{\infty}} \geq \min _{i \in I}\left|u_{i}^{\diamond}\right| \frac{1}{\left\|\left(P_{I} K^{*} K P_{I}\right)^{-1}\right\|_{\ell^{1}, \ell^{1}}} .
$$

Proof of theorem 2.3. For the deduction of condition (2.2) from condition (2.1), one can split the operator $P_{I} K^{*} K P_{I}$ into diagonal and off-diagonal and use Neumann series expansion for its inverse, following the techniques from [11,6]. A comprehensive proof can be found in [19].

4. Conclusion and future prospects. We have obtained two conditions that ensure exact recovery of the support of the unknown solution $u^{\diamond}$. Surprisingly the conditions for exact recovery given here are valid for both methods, for the Tikhonov regularization with a sparsity enforcing $\ell^{1}$ penalty and for the orthogonal matching pursuit, although the proofs are done differently. This resembles what is known for the noiseless case, see [20] and [7], respectively.

Hence, a further direction of research may be to investigate other types of algorithms with sparsity enhancing properties, as e.g. the basis pursuit [2], other iterative greedy algorithms $[17,16]$ or the Tikhonov functional with sublinear $\ell^{p}$ penalty term $[1,9,22]$. Possibly, the the $\varepsilon$ ERC (2.1) and the Neumann $\varepsilon$ ERC (2.2) turn out to be valid for other methods incorporating sparsity constraints. 


\section{REFERENCES}

[1] K. Bredies And D. A. Lorenz, Regularization with non-convex separable constraints, Inverse Problems, 25:8 (2009), p. 085011 (14pp).

[2] S. S. Chen, D. L. Donoho, and M. A. Saunders, Atomic decomposition by basis pursuit, SIAM Journal on Scientific Computing, 20:1 (1998), pp. :33-61.

[3] I. Daubechies, M. Defrise, and C. De Mol, An iterative thresholding algorithm for linear inverse problems with a sparsity constraint, Communications in Pure and Applied Mathematics, 57:11, pp. :1413-1457.

[4] L. Denis, D. A. Lorenz, And D. Trede, Greedy solution of ill-posed problems: Error bounds and exact inversion, Inverse Problems, 25:11 (2009), pp. 115017 (24pp).

[5] D. L. Donoho, M. Elad, and V. Temlyakov, Stable recovery of sparse overcomplete representations in the presence of noise, IEEE Transactions on Information Theory, 52:1 (2006), pp. 6-18.

[6] C. Dossal and S. Mallat, Sparse spike deconvolution with minimum scale, in Proceedings of the First Workshop "Signal Processing with Adaptive Sparse Structured Representations", 2005.

[7] J.-J. FuCHS, On sparse representations in arbitrary redundant bases, IEEE Transactions on Information Theory, 50:6 (2004), pp. 1341-1344.

[8] J.-J. Fuchs, Recovery of exact sparse representations in the presence of bounded noise, IEEE Transactions on Information Theory, 51:10 (2005), pp. 3601-3608.

[9] M. Grasmair, Well-posedness and convergence rates for sparse regularization with sublinear $l^{q}$ penalty term, Inverse Problems and Imaging, 3:3 (2009), pp. 383-387.

[10] M. Grasmair, M. Haltmeier, and O. Scherzer, Sparse regularization with $\ell^{q}$ penalty term, Inverse Problems, 24:5, p. 055020 (13pp).

[11] R. Gribonval and M. Nielsen, Beyond sparsity: Recovering structured representations by $\ell^{1}$ minimization and greedy algorithms, Advances in Computational Mathematics, 28:1 (2008), pp. :23-41.

[12] D. A. Lorenz, Convergence rates and source conditions for Tikhonov regularization with sparsity constraints, Journal of Inverse and Ill-Posed Problems, 16:5 (2008), pp. 463-478.

[13] D. A. Lorenz, S. Schiffler, And D. Trede, Beyond convergence rates: Exact inversion with Tikhonov regularization with sparsity constraints, arXiv:1001.3276, 2010.

[14] I. LORIS, On the performance of algorithms for the minimization of $\ell^{1}$-penalized functionals, Inverse Problems, 25:3 (2009), p. 035008 (16pp).

[15] S. Mallat, G. Davis, And Z. Zhang, Adaptive time-frequency decompositions, SPIE Journal of Optical Engineering, 33:7 (1994), pp. :2183-2191.

[16] D. NeEdell ANd J. A. TROpP, CoSaMP: Iterative signal recovery from incomplete and inaccurate samples, Applied and Computational Harmonic Analysis, 26:3 (2009), pp. 301-321.

[17] D. NeEdell AND R. Vershynin, Uniform uncertainty principle and signal recovery via regularized orthogonal matching pursuit, Foundations of Computational Mathematics, 9:3 (2009), pp. 317-334.

[18] Y. PATI, R. REZAIIFAR, AND P. KRISHNAPRASAD, Orthogonal matching pursuit: recursive function approximation with applications to wavelet decomposition, in Proceedings of 27 th Asilomar Conference on Signals, Systems and Computers, volume 1, pp. 40-44, 1993.

[19] D. Trede, Inverse Problems with Sparsity Constraints: Convergence Rates and Exact Recovery, PhD thesis, Univerität Bremen, 2010.

[20] J. A. Tropp, Greed is good: Algorithmic results for sparse approximation, IEEE Transactions on Information Theory, 50:10 (2004), pp. 2231-2242.

[21] J. A. Tropp, Just relax: Convex programming methods for identifying sparse signals in noise, IEEE Transactions on Information Theory, 52:3 (2006), pp. 1030-1051.

[22] C. A. ZARZER, On Tikhonov regularization with non-convex sparsity constraints, Inverse Problems, 25:2 (2009), p 025006 (13pp). 